\title{
Evaluation of Density Matrix and Helmholtz Free Energy for Harmonic Oscillator Asymmetric Potential via Feynmans Approach
}

\author{
Piyarut Moonsri ${ }^{1}$ and Artit Hutem ${ }^{2}$ \\ ${ }^{1}$ Chemistry Division, Faculty of Science and Technology, Phetchabun Rajabhat University, Phetchabun 67000, Thailand \\ ${ }^{2}$ Physics Division, Faculty of Science and Technology, Phetchabun Rajabhat University, Phetchabun 67000, Thailand \\ Correspondence should be addressed to Artit Hutem; magoohootem@yahoo.com
}

Received 18 August 2014; Revised 8 October 2014; Accepted 9 October 2014; Published 2 November 2014

Academic Editor: Miquel Solà

Copyright ( $\odot 2014$ P. Moonsri and A. Hutem. This is an open access article distributed under the Creative Commons Attribution License, which permits unrestricted use, distribution, and reproduction in any medium, provided the original work is properly cited.

We apply a Feynmans technique for calculation of a canonical density matrix of a single particle under harmonic oscillator asymmetric potential and solving the Bloch equation of the statistical mechanics system. The density matrix $\left(\widehat{\mathscr{P}}_{u}\right)$ and kinetic energy per unit length $\left(\widehat{\tau}_{L}\right)$ can be directly evaluated from the solving solutions. From the evaluation, it was found that both of the density matrix and kinetic energy per unit length depended on the parameter of the value of asymmetric potential $(\lambda)$, the value of axes-shift potential $(g)$, and temperature $(T)$. Comparison of the Helmholtz free energy was derived by the Feynmans technique and the path-integral method. The results illustrated are slightly different.

\section{Introduction}

We consider a quantum mechanical ensemble of identical systems in situations where the description is incomplete. When the description of the system is incomplete, the state of the system is described by means of what is called a density matrix. In the statistical point of view, a state of quantum ensemble is described by a density operator (or statistical operator) $\widehat{\mathscr{P}}_{u}$; the corresponding matrix is called a density matrix. A knowledge of this matrix enables us to calculate the mean value of physical quantity of the system and also the probabilities of various values of such a quantity.

March and Murray used the Bloch equation to calculate the canonical density matrix in a single particle framework which may be related directly to the generalized canonical density matrix, containing the Fermi-Dirac function [1]. Howard et al. used the Bloch equation evaluate the electron density $\widehat{\mathscr{P}}_{u}$ and kinetic energy $\widehat{\tau}_{L}(x)$ for the one dimensional $\operatorname{sech}^{2}(x)$ potential. Howard et al. used tool employed is the Slater sum, which satisfies a partial differential equation [2].

For calculation of the canonical density matrix in terms of the Slater sum and kinetic energy per unit length in terms of the Slater sum see March and Nieto [3]. To study problems of the canonical ensemble, we focus on the calculation the canonical density matrix and the Helmholtz free energy of a single particle under asymmetric harmonic oscillator potential.

The scheme of the paper is as follows. In Section 2, there is detailing with the density matrix. In Section 3, we show the calculation of the canonical density matrix (or charge bondorder matrix) and the Helmholtz free energy by Feynmans approach. In Section 4, we representation of the evaluation of the density matrix and the Helmholtz free energy by the path-integral method $(\kappa(y))$ method. In Section 5 we make a presentation of numerical results for the Helmholtz free energy of a single particle under asymmetric harmonic oscillator potential. The conclusion and discussion are given in Section 6.

\section{Statistical Density Matrix}

A density matrix is a matrix that describes a quantum system in a mixed state, a statistical ensemble of several quantum states. This should be contrasted with a single state 
vector that describes a quantum system in a pure state. The density matrix is the quantum-mechanical analogue to a phase-space probability measure (probability distribution of position and momentum) in classical statistical mechanics. Explicitly, suppose a quantum system may be found in state $\left|\psi_{1}\right\rangle$ with probability $P_{1}$ or it may be found in state $\left|\psi_{2}\right\rangle$ with probability $P_{2}$ or it may be found in state $\left|\psi_{3}\right\rangle$ with probability $P_{3}$, and so on. The density matrix for this system is

$$
\widehat{\mathscr{P}}=\sum_{n} P_{n}\left|\psi_{n}\right\rangle\left\langle\psi_{n}\right|,
$$

where $\left(\left|\psi_{n}\right\rangle\right)$ need not be orthogonal and $\sum_{n} P_{n}=1$. By choosing an orthonormal basis $\left(\left|u_{j}\right\rangle\right)$, one may resolve the density operator into the density matrix, whose elements are

$$
\widehat{\mathscr{P}}_{i, j}=\sum_{n} P_{n}\left\langle u_{j} \mid \psi_{n}\right\rangle\left\langle\psi_{n} \mid u_{i}\right\rangle
$$

For an operator $\widehat{\mathscr{A}}$ (which describes an observable $\widehat{\mathscr{A}}$ of the system), the expectation value $\langle\widehat{\mathscr{A}}\rangle$ is given by

$$
\langle\widehat{\mathscr{A}}\rangle=\sum_{n} P_{n}\left\langle\psi_{n}|\widehat{\mathscr{A}}| \psi_{n}\right\rangle=\sum_{i}\left\langle u_{i}|\widehat{\mathscr{P}} \widehat{\mathscr{A}}| u_{i}\right\rangle=\operatorname{tr}(\widehat{\mathscr{P}} \widehat{\mathscr{A}}) .
$$

In the canonical ensemble, the probability that the system is in state $|n\rangle$ is given by

$$
P_{n}=\frac{e^{-\beta \mathscr{E}_{n}}}{\sum_{n} e^{\beta \mathscr{E}_{n}}}
$$

and the thermal average of an arbitrary operator $\widehat{\mathscr{A}}$ is

$$
\begin{aligned}
\langle\widehat{\mathscr{A}}\rangle & =\sum_{n}\langle n|\widehat{\mathscr{A}}| n\rangle\left(\frac{e^{-\beta \mathscr{E}_{n}}}{\sum_{n} e^{\beta \mathscr{E}_{n}}}\right) \\
& =\frac{1}{\widehat{Q}_{N}(\beta)} \sum_{n}\langle n|\widehat{\mathscr{A}}| n\rangle e^{-\beta \mathscr{E}_{n}} .
\end{aligned}
$$

The operator

$$
\widehat{\mathscr{P}}=\frac{1}{\widehat{Q}_{N}(\beta)} \sum_{n}|n\rangle\langle n| e^{-\beta \mathscr{E}_{n}}
$$

is commonly referred to as density operator and its matrix representation as the density matrix. Here, $Q_{N}(\beta)$ is the partition function.

\section{A Linear Harmonic Oscillator Asymmetric Potential by Feynmans Approach}

Next, we consider the case of a linear harmonics oscillator of asymmetric potential, whose Hamiltonian is given by [4]

$$
\widehat{\mathscr{H}}_{S}=\frac{\widehat{P}^{2}}{2 m}+\frac{1}{2} m \omega^{2} \widehat{x}^{2}+\sqrt{\frac{m \omega}{\hbar}} \frac{\hbar \omega}{2} \lambda\left(\widehat{x}-g \sqrt{\frac{\hbar}{m \omega}}\right) ;
$$

we note that this is a time-independent Hamiltonian of harmonic oscillator asymmetric axes-shift potential system.
Substituting in the time-independent Hamiltonian equation (7) into the Bloch equation [3], we can rewrite the differential equation completely in terms of $\widehat{\mathscr{P}}_{u}(\xi, \xi, f)$ as

$$
\begin{aligned}
-\frac{\partial \widehat{\mathscr{P}}_{u}}{\partial \beta}= & -\frac{\hbar^{2}}{2 m} \frac{\partial^{2} \widehat{\mathscr{P}}_{u}}{\partial x^{2}}+\frac{1}{2} m \omega^{2} \widehat{x}^{2} \widehat{\mathscr{P}}_{u} \\
& +\sqrt{\frac{m \omega}{\hbar}} \frac{\hbar \omega}{2} \lambda\left(\widehat{x}-g \sqrt{\frac{\hbar}{m \omega}}\right) \widehat{\mathscr{P}}_{u} .
\end{aligned}
$$

We define some new dimensionless variable. The position variable $\widehat{x}=x$ is replaced with the dimensionless variable $\xi$

$$
\xi=\sqrt{\frac{m \omega}{\hbar}} x, \quad x^{2}=\frac{\hbar}{m \omega} \xi^{2}, \quad \beta=\frac{2 f}{\hbar \omega} .
$$

Substituting in for $x$ in terms of $\xi$, we can rewrite the differential equation completely in terms of $\xi$ and $\widehat{\mathscr{P}}_{u}$ as

$$
-\frac{\partial \widehat{\mathscr{P}}_{u}}{\partial f}=-\frac{\partial^{2} \widehat{\mathscr{P}}_{u}}{\partial \xi^{2}}+\xi^{2} \widehat{\mathscr{P}}_{u}+\lambda \xi \widehat{\mathscr{P}}_{u}-\lambda g \widehat{\mathscr{P}}_{u} .
$$

Knowing the Gaussian dependence of the density matrix $\widehat{\mathscr{P}}_{u}$ in case of the free particle, we try the following form:

$$
\begin{gathered}
\widehat{\mathscr{P}}_{u}(\xi, \dot{\xi}, f) \approx e^{-\left(a(f) \xi^{2}+b(f) \xi+c(f)\right)}, \\
\lambda g \widehat{\mathscr{P}}_{u}=\lambda g e^{-\left(a(f) \xi^{2}+b(f) \xi+c(f)\right)}, \\
-\frac{\partial \widehat{\mathscr{P}}_{u}}{\partial f}=\left[\xi^{2} \frac{d a(f)}{d f}+\xi \frac{d b(f)}{d f}+\frac{d c(f)}{d f}\right] \\
\times e^{-\left(a(f) \xi^{2}+b(f) \xi+c(f)\right)}, \\
-\frac{\partial^{2} \widehat{\mathscr{P}}_{u}}{\partial \xi^{2}}=\left(2 a(f)-4 a^{2}(f) \xi^{2}-4 a(f) b(f) \xi-b^{2}(f)\right) \\
\times e^{-\left(a(f) \xi^{2}+b(f) \xi+c(f)\right)}, \\
\xi^{2} \widehat{\mathscr{P}}_{u}=\xi^{2} e^{-\left(a(f) \xi^{2}+b(f) \xi+c(f)\right)}, \\
\lambda \xi \widehat{\mathscr{P}}_{u}=\lambda \xi e^{-\left(a(f) \xi^{2}+b(f) \xi+c(f)\right)} .
\end{gathered}
$$

Substituting expansion equation (11) into (10) leads us to the equation

$$
\begin{aligned}
\frac{d a(f)}{d f} & \xi^{2}+\frac{d b(f)}{d f} \xi+\frac{d c(f)}{d f} \\
= & \left(1-4 a^{2}(f)\right) \xi^{2}+(\lambda-4 a(f) b(f)) \xi \\
& +\left(2 a(f)-\lambda g-b^{2}(f)\right) .
\end{aligned}
$$


Equating the coefficients for $\xi^{2}, \xi$, and $\xi^{0}=1$ on both sides of the last equation (12) gives

$$
\begin{gathered}
\frac{d a(f)}{d f}=\left(1-4 a^{2}(f)\right), \\
\frac{d b(f)}{d f}=(\lambda-4 a(f) b(f)), \\
\frac{d c(f)}{d f}=\left(2 a(f)-\lambda g-b^{2}(f)\right),
\end{gathered}
$$

respectively. Computing (13) is integrated to give

$$
a(f)=\frac{1}{2} \operatorname{coth}(2 f) \text {. }
$$

Equation (14) is the linear first-order ordinary differential equation to give $[5,6]$

$$
\begin{gathered}
\lambda=\frac{d b(f)}{d f}+2 \operatorname{coth}(2 f) b(f), \\
b(f)=e^{-\int 2 \operatorname{coth}(2 f) d f}\left(\int \lambda e^{\int 2 \operatorname{coth}(2 f) d f} d f+A\right), \\
b(f)=\frac{\lambda}{2} \operatorname{coth}(2 f)+A \operatorname{csch}(2 f) .
\end{gathered}
$$

Equation (15) is integrated with respect to $f$ as

$$
\begin{gathered}
\frac{d c(f)}{d f}=\left(\operatorname{coth}(2 f)-\lambda g-\left(\frac{\lambda}{2} \operatorname{coth}(2 f)+A \operatorname{csch}(2 f)\right)^{2}\right) \\
c(f)=\int \operatorname{coth}(2 f) d f-\int \lambda g d f \\
-\int\left(\frac{\lambda}{2} \operatorname{coth}(2 f)+A \operatorname{csch}(2 f)\right)^{2} d f \\
c(f)=\frac{1}{8}\left(-2 f \lambda(4 g+\lambda)+\left(4 A^{2}+\lambda^{2}\right) \operatorname{coth}(2 f)\right. \\
+4 \ln (\sinh (2 f)) \\
+2 A \lambda \operatorname{csch}(f) \operatorname{sech}(f)-\ln B)
\end{gathered}
$$

where $B, \lambda$, and $g$ are constants. Substituting $a(f), b(f)$, and $c(f)$ of (16), (17), and (18), respectively, into (11), we may rewrite density matrix as

$\widehat{\mathscr{P}}_{u}$

$$
\begin{aligned}
= & \frac{B}{\sqrt{\sinh (2 f)}} \\
& \times \exp \left(-\left[\left(\frac{\xi^{2}}{2}+\frac{\xi \lambda}{2}+\frac{1}{8}\left(4 A^{2}+\lambda^{2}\right)\right) \operatorname{coth}(2 f)\right.\right. \\
& \left.\left.+\left(\xi A+\frac{A \lambda}{2}\right) \operatorname{csch}(2 f)-\frac{f \lambda}{4}(4 g+\lambda)\right]\right) .
\end{aligned}
$$

Thus, the Taylor series expansion of $\sinh (2 f), \operatorname{coth}(2 f)$ about $f \rightarrow 0$ is given by

$$
\lim _{f \rightarrow 0} \sinh (2 f) \approx 2 f, \quad \lim _{f \rightarrow 0} \operatorname{coth}(2 f) \approx \frac{1}{2 f} ;
$$

we obtain the density matrix final form:

$$
\begin{aligned}
& \widehat{\mathscr{P}}_{u} \approx \frac{B}{\sqrt{2 f}} \\
& \times \exp \left(-\left[\left(\frac{\xi^{2}}{2}+\frac{\xi \lambda}{2}+\frac{1}{8}\left(4 A^{2}+\lambda^{2}\right)\right) \frac{1}{2 f}\right.\right. \\
&\left.\left.+\left(\xi A+\frac{A \lambda}{2}\right) \frac{1}{2 f}-\frac{\lambda f}{4}(4 g+\lambda)\right]\right) .
\end{aligned}
$$

The initial condition is

$$
\widehat{\mathscr{P}}_{u}=\delta(x-\dot{x}) \quad \text { for } f=0
$$

or

$$
\widehat{\mathscr{P}}_{u}=\sqrt{\frac{m \omega}{\hbar}} \delta(\xi-\xi) \quad \text { for } f=0
$$

For low $f=(\hbar \omega / 2) \beta=\hbar \omega / 2 k_{B} T$ (high temperature) the particle should act almost like a free particle, as its probable kinetic energy is so high. Therefore, we expect that, for low $f$, the density matrix for a harmonic oscillator will be given approximately by [7]

$$
\widehat{\mathscr{P}}_{u f r e}(\xi, \dot{\xi}, f) \approx \sqrt{\frac{m \omega}{4 \pi \hbar f}} e^{-(\xi-\xi)^{2} / 4 f} .
$$

Comparing the value of $A, B$ in (21) with (24) identifies the solution as

$$
\frac{B}{\sqrt{2 f}}=\sqrt{\frac{m \omega}{4 \pi \hbar f}} \longrightarrow B=\sqrt{\frac{m \omega}{2 \pi \hbar}},
$$

$$
\begin{aligned}
\xi^{2}-2 \xi \dot{\xi}+\dot{\xi}^{2} & \\
= & \xi^{2}+(\lambda+2 A) \xi \\
+ & \left(A^{2}+\frac{\lambda^{2}}{4}+A \lambda-4 g \lambda f^{2}-\lambda^{2} f^{2}\right), \\
A & =-\left(\xi+\frac{\lambda}{2}\right) .
\end{aligned}
$$

Substituting (25) into (19) and setting $\xi=\dot{\xi}$ produce

$$
\begin{aligned}
\widehat{\mathscr{P}}_{u}(\xi, f) \approx & \sqrt{\frac{m \omega}{2 \pi \hbar \sinh (2 f)}} \\
& \times e^{-\left[(\xi+(\lambda / 2))^{2}((\cosh (2 f)-1) / \sinh (2 f))-(\lambda f / 4)(\lambda+4 g)\right]} .
\end{aligned}
$$

Using the identity of trigonometric $\tanh (x / 2)=$ $(\cosh (x)-1) / \sinh (x)$ and setting $\xi=\sqrt{m \omega / \hbar} x$ into 
(26), we can rewrite the canonical density matrix or charge bond-order matrix [8] as

$$
\begin{aligned}
\widehat{\mathscr{P}}_{u}(x, f) \approx & \sqrt{\frac{m \omega}{2 \pi \hbar \sinh (2 f)}} \\
& \times e^{-(m \omega / 4 \hbar)\left[(2 x+\sqrt{\hbar / m \omega} \lambda)^{2} \tanh (f)-(\lambda f \hbar / m \omega)(\lambda+4 g)\right]} .
\end{aligned}
$$

The obtained results are neatly summarized in Figure 1. It is the probability density for finding the system at $x$; we note as a Gaussian distribution in $x$. Let us define four new the variables $\gamma, \eta, \mu$, and $\phi$ as function of $f$ by

$$
\begin{gathered}
\gamma(f)=\sqrt{\frac{m \omega}{2 \pi \hbar \sinh (2 f)}}, \quad \eta(f)=\frac{m \omega}{4 \hbar} \tanh (f), \\
\mu(f)=\frac{\lambda f}{4}(\lambda+4 g), \quad \phi=\sqrt{\frac{\hbar}{m \omega} \lambda ;}
\end{gathered}
$$

we can rewrite the density matrix completely in terms of the new variable $(\gamma, \eta, \mu, \phi)$ as

$$
\widehat{\mathscr{P}}_{u}(x, f) \approx \gamma e^{-\left(\eta(2 x+\phi)^{2}-\mu\right)} .
$$

The expectation value of $x^{2}$ in the density matrix $\widehat{\mathscr{P}}_{u}(x, f)$ can be determined from the definition of the expectation values as

$$
\begin{aligned}
\left\langle x^{2}\right\rangle & =\frac{\int_{-\infty}^{\infty} \widehat{x}^{2} \widehat{\mathscr{P}}_{u}(x, f) d x}{\int_{-\infty}^{\infty} \widehat{\mathscr{P}}_{u}(x, f) d x} \\
& =\frac{\gamma \sqrt{\pi} e^{\mu}}{16 \eta^{3 / 2}} \frac{2 \sqrt{\eta}}{\gamma \sqrt{\pi} e^{\mu}}\left(1+2 \eta \phi^{2}\right), \\
\left\langle x^{2}\right\rangle & =\frac{\hbar}{2 m \omega} \operatorname{coth}\left(\frac{\hbar \omega \beta}{2}\right)+\frac{\hbar}{4 m \omega} \lambda^{2} .
\end{aligned}
$$

The average of the potential energy $\left(E_{s}\right)$ is thus

$$
\begin{gathered}
\left\langle E_{s}\right\rangle=\frac{1}{2} m \omega^{2}\left\langle x^{2}\right\rangle \\
\left\langle E_{s}\right\rangle=\frac{\hbar \omega}{4} \operatorname{coth}\left(\frac{\hbar \omega \beta}{2}\right)+\frac{\hbar \omega}{8} \lambda^{2} .
\end{gathered}
$$

The kinetic energy per unit length $\widehat{\tau}_{L}(x, f)$ is defined from the canonical density matrix $\widehat{\mathscr{P}}_{u}(x, f)$ as [3]

$$
\widehat{\tau}_{L}(x, f)=-\frac{\hbar^{2}}{2 m} \frac{\partial^{2}}{\partial x^{2}} \widehat{\mathscr{P}}_{u}(x, f) .
$$

By substituting (29) into (32), we can write the kinetic energy per unit length completely as follows

$$
\begin{aligned}
\widehat{\tau}_{L}(x, T)= & \frac{\hbar \omega}{2} \tanh \left(\frac{\hbar \omega}{2 k_{B} T}\right) \sqrt{\frac{m \omega}{2 \pi \hbar \sinh \left(\hbar \omega / k_{B} T\right)}} \\
& \times e^{\left(\lambda \hbar \omega / 8 k_{B} T\right)(\lambda+4 g)} \\
& \times\left[2-\frac{m \omega}{\hbar} \tanh \left(\frac{\hbar \omega}{2 k_{B} T}\right)\left(2 x+\sqrt{\frac{\hbar}{m \omega} \lambda}\right)^{2}\right] \\
& \times e^{-(m \omega / 4 \hbar) \tanh \left(\hbar \omega / 2 k_{B} T\right)(2 x+\sqrt{\hbar / m \omega} \lambda)^{2}} .
\end{aligned}
$$

The obtained results from (33) are summarized in Figure 2.

Now, the Helmholtz free energy of the harmonic oscillator asymmetric potential system $\left(F_{\mathrm{New}}\right)$ is given by

$$
F_{\mathrm{New}}=-\frac{1}{\beta} \ln Q_{N}(\beta)
$$

or

$$
\widehat{Q}_{N}(\beta)=e^{-\beta F_{\text {New }}} \text {. }
$$

The density matrix of the system in the integration representation will be given by

$$
e^{-\beta F_{\text {new }}}=\int_{-\infty}^{\infty} \widehat{\mathscr{P}}_{u}(x, \beta) d x .
$$

Substituting (29) into (36), we obtain for the partition function of the particle in harmonic oscillator asymmetric potential system

$$
\begin{aligned}
e^{-\beta F_{\text {new }}} & =\int_{-\infty}^{\infty} \gamma e^{-\left(\eta(2 x+\phi)^{2}-\mu\right)} d x \\
& =\frac{\gamma}{2} \sqrt{\frac{\pi}{\eta}} \\
& =\frac{e^{(\lambda f / 4)(\lambda+4 g)}}{2 \sinh (f)} .
\end{aligned}
$$

Thus, we have the following results:

$$
F_{\text {new }}=\frac{1}{\beta} \ln (2 \sinh (f))-\frac{\lambda f}{4 \beta}(\lambda+4 g) .
$$

This is the Helmholtz free energy for a one-dimensional harmonic oscillator asymmetric potential $\left(F_{\text {new }}\right)$, as already derived. When we write the Helmholtz free energy in new variable is $G_{\text {new }}=2 F_{\text {new }} / \hbar \omega, f=\hbar \omega \beta / 2$ into (38), we obtain that

$$
G_{\text {new }}=\frac{1}{f} \ln (2 \sinh (f))-\frac{\lambda}{4}(\lambda+4 g) .
$$

One may summarize the numerical results of Helmholtz free energy for harmonic oscillator asymmetric potential by 


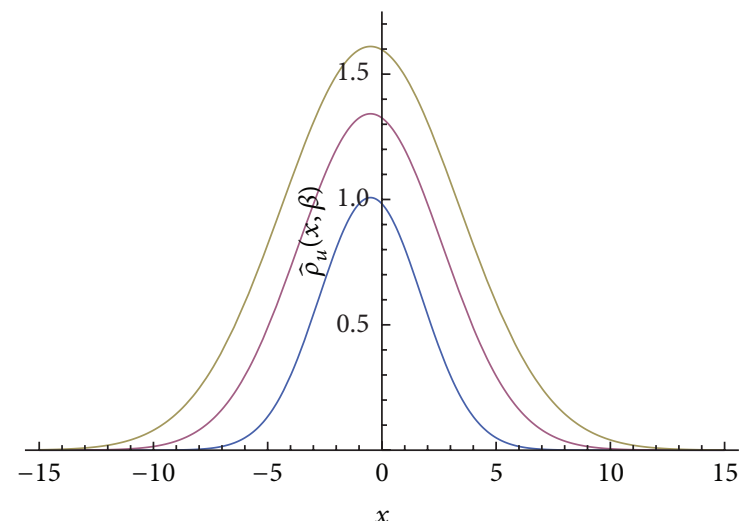

$\begin{aligned} T_{1} & =5 \mathrm{~K} \\ -T_{2} & =10 \mathrm{~K} \\ T_{3} & =15 \mathrm{~K}\end{aligned}$

(a) Density Matrix, $\lambda=1, g=1$

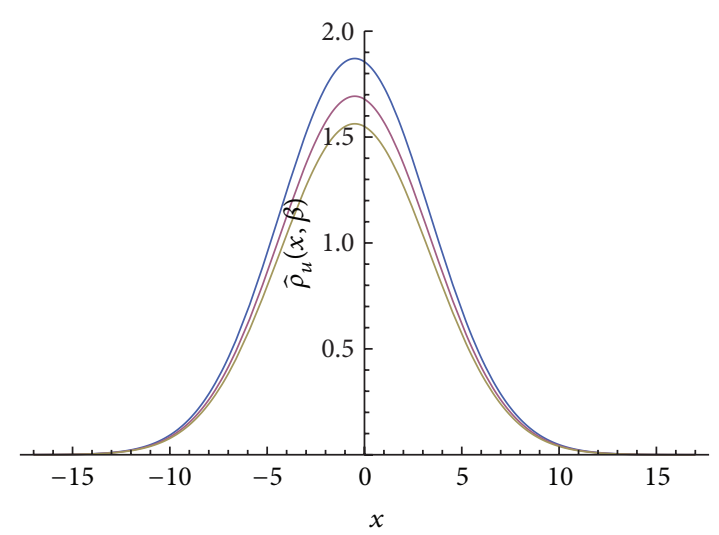

$\begin{aligned}-g_{1} & =0.1 \\ - & g_{2}=2.5 \\ -g_{3} & =5.5\end{aligned}$

(c) Density Matrix, $\lambda=1, g=+, T=15$

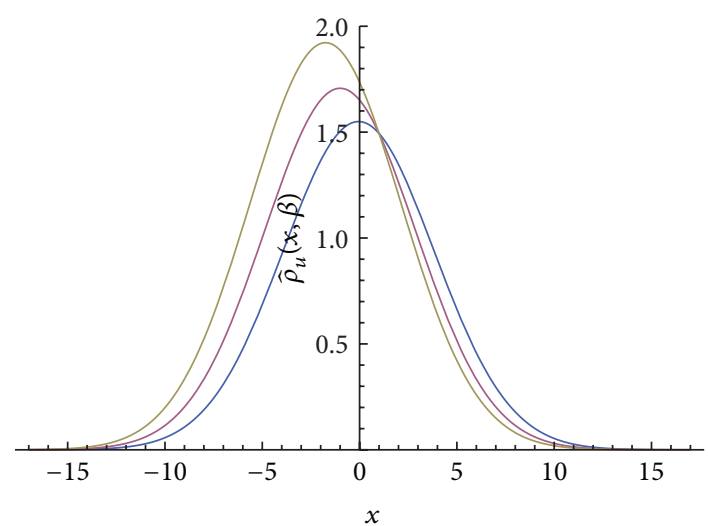

$-\lambda_{1}=0.1$

$-\lambda_{2}=2.0$

$-\lambda_{3}=3.5$

(b) Density Matrix, $g=1, T=15$

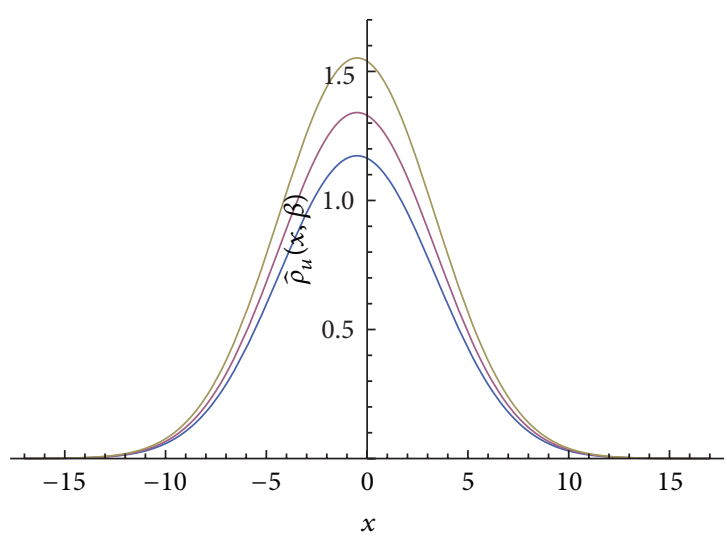

$\begin{aligned}-g_{1} & =-0.1 \\ -g_{2} & =-4.5 \\ -g_{3} & =-8.5\end{aligned}$

(d) Density Matrix, $\lambda=1, g=+, T=15$

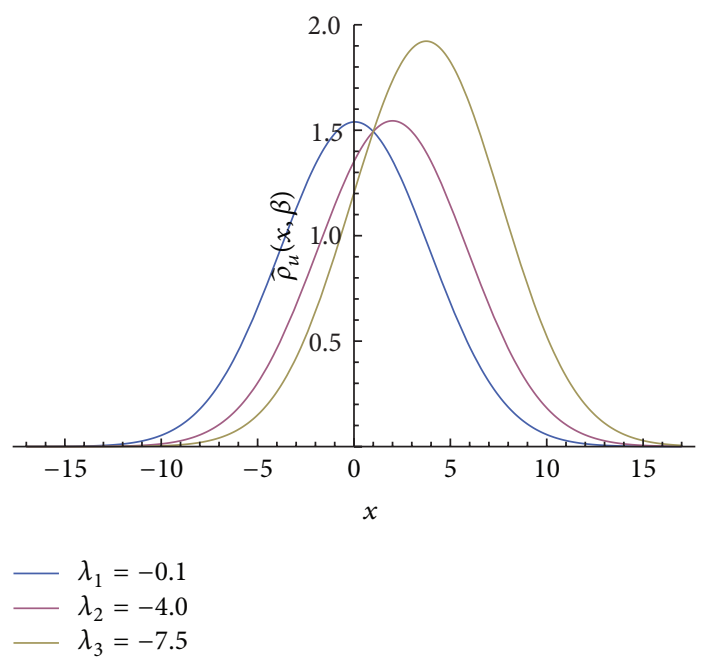

(e) Density Matrix, $g=1, T=15$

FIGURE 1: Plot of the canonical density matrix of a single particle for harmonic oscillator asymmetric potential system. 

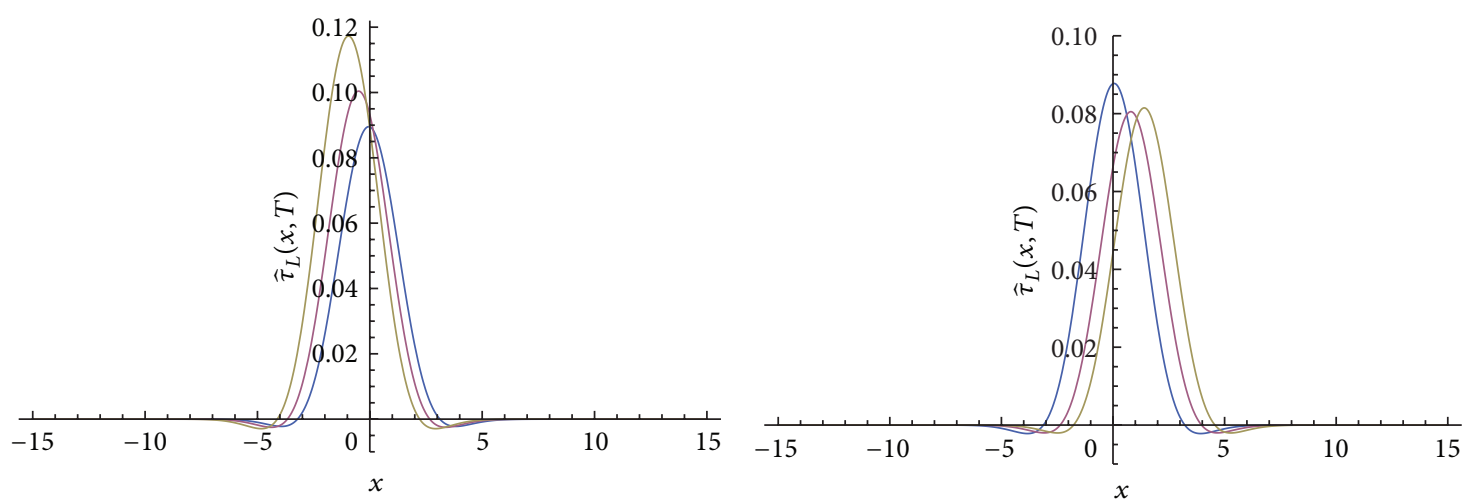

$$
\begin{aligned}
-\lambda_{1} & =0.1 \\
-\lambda_{2} & =1.0 \\
-\lambda_{3} & =1.9
\end{aligned}
$$

$$
\begin{aligned}
\lambda_{1} & =-0.1 \\
\lambda_{2} & =-1.6 \\
\lambda_{3} & =-2.8
\end{aligned}
$$

(a) Kinetic Energy, $g=1, T=5 \mathrm{~K}$

(b) Kinetic Energy, $g=1, T=5 \mathrm{~K}$
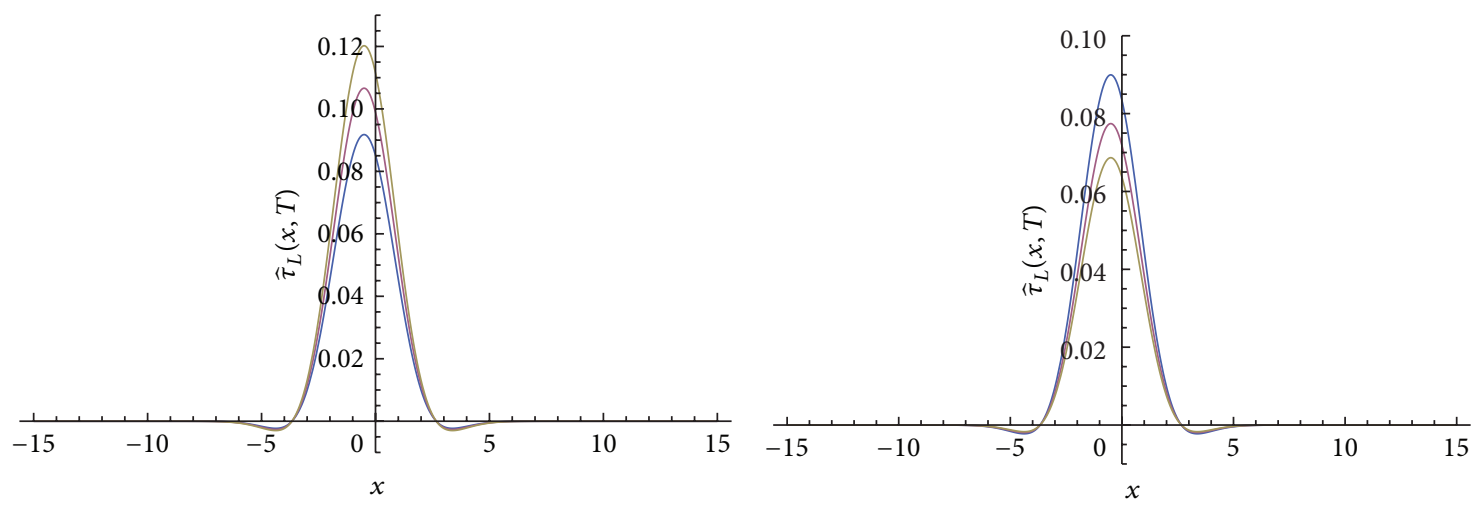

$\begin{aligned}-g_{1} & =0.1 \\ -g_{2} & =1.6 \\ -g_{3} & =2.8\end{aligned}$

$-g_{1}=-0.1$

$-g_{2}=-1.6$

- $g_{3}=-2.8$

(c) Kinetic Energy, $\lambda=1, T=5 \mathrm{~K}$

(d) Kinetic Energy, $\lambda=1, T=5 \mathrm{~K}$

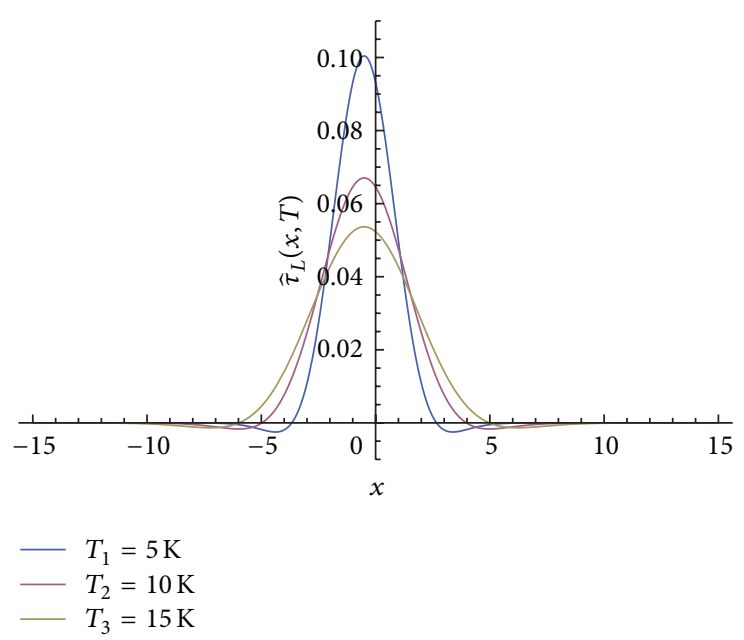

(e) Kinetic Energy, $\lambda=1, g=1$

FIGURE 2: Illustration the kinetic energy per unit length under harmonic oscillator asymmetric potential system. 

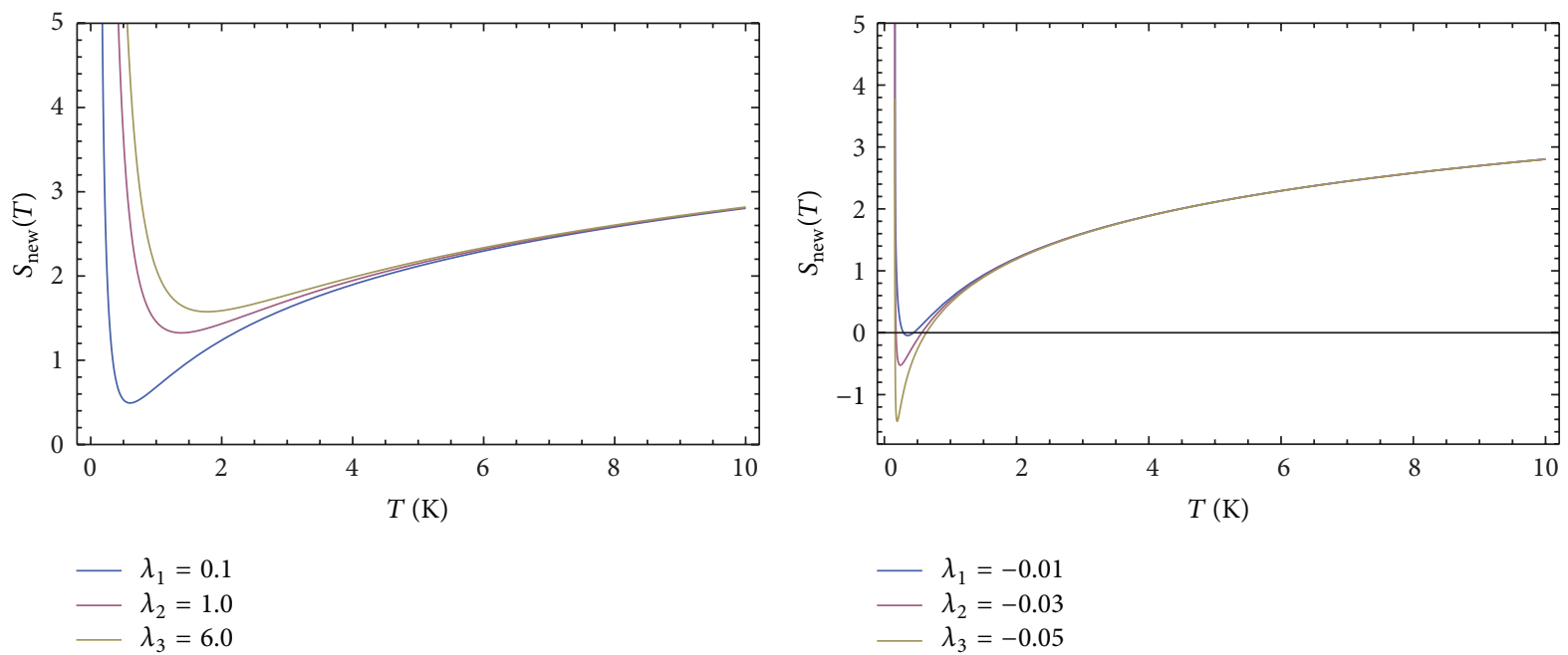

(a) $\operatorname{Entropy}\left(S_{\text {new }}(T)\right), g=-2$

(b) Entropy $\left(S_{\text {new }}(T)\right), g=-4$

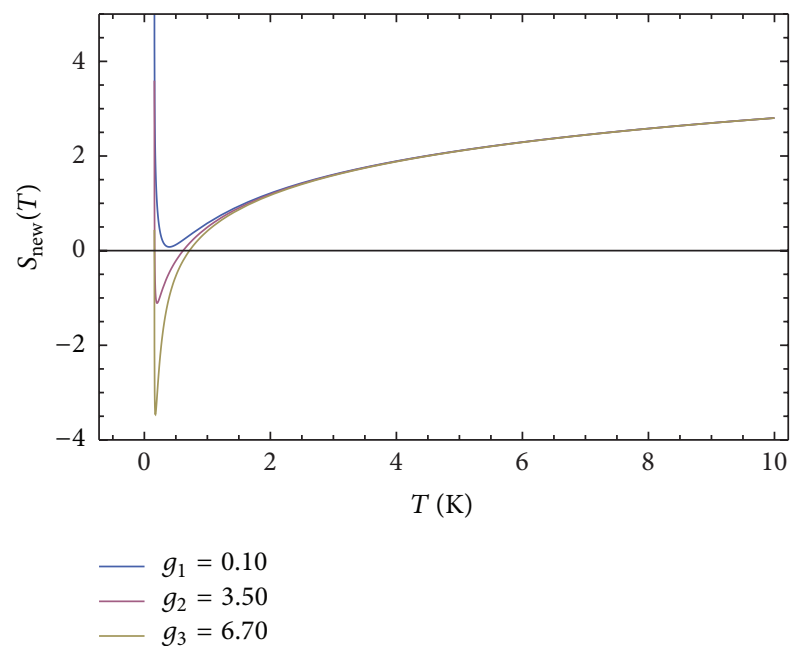

(c) $\operatorname{Entropy}\left(S_{\text {new }}(T)\right), \lambda=0.05$

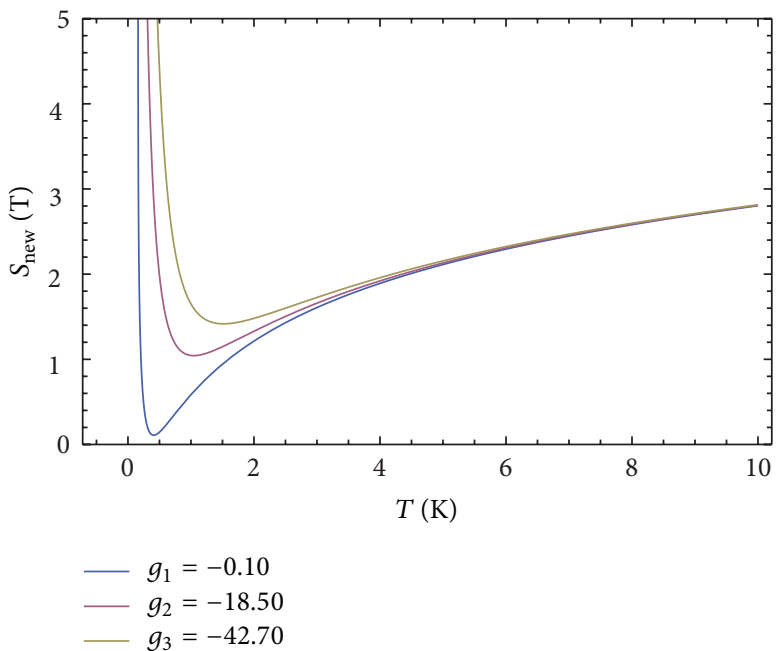

(d) Entropy $\left(S_{\text {new }}(T)\right), \lambda=0.05$

FIGURE 3: Illustration of the entropy $(S)$ of a single particle under harmonic oscillator asymmetric potential.

Feynmans approach in Section 5. In Section 5, comparison of Helmholtz free energy for harmonic oscillator asymmetric potential obtain from Feynmans approach and the pathintegral method $\kappa(y)$. This work aims at computing the entropy $(S)$ of the harmonic oscillator asymmetric potential using the Helmholtz free energy in (38). Using the relation

$$
S_{\text {new }}(T)=-\frac{\partial F_{\text {new }}(T)}{\partial T}=k_{B}\left(\frac{\partial}{\partial T}\left(T \ln \left(Q_{N}(\beta)\right)\right)\right)
$$

the entropy of a system can be write as

$$
\begin{aligned}
S_{\text {new }}(T)= & \frac{k_{B} \hbar \omega}{4\left(k_{B} T\right)} \frac{\cosh \left(\hbar \omega / 2 k_{B} T\right)}{\sinh \left(\hbar \omega / 2 k_{B} T\right)} \\
& -k_{B} \ln \left(2 \sinh \left(\hbar \omega / 2 k_{B} T\right)\right) \\
& -\frac{k_{B} \lambda \omega \hbar}{8\left(k_{B} T\right)^{2}}(\lambda+4 g) .
\end{aligned}
$$

Equation (41) is plotted in Figure 3(a) assuming the value of axes-shift potential $g$ equal -2 and vary the value of asymmetric potential $\lambda$. From (41) are plotted in Figure 3 assuming the value of asymmetric potential $\lambda$ equal 0.05 and vary the value of axes-shift potential $g$. Figure 3 shows that, as $\lambda$ increases, the depth of the entropy decreases and its minimum moves farther away the origin.

\section{Evaluate Helmholtz Free Energy via Path- Integral Method $(\kappa(y))$}

In the Helmholtz free energy the quantity of the thermodynamics of a given harmonic oscillator asymmetric potential system is derived from its the path-integral method:

$$
\kappa(y)=\sqrt{\frac{6 m k_{B} T}{\pi \hbar^{2}}} \int_{-\infty}^{\infty} e^{-\left(6 m k_{B} T(y-z)^{2} / \hbar^{2}\right)} V(z) d z .
$$


In this case of the harmonic oscillator asymmetric potential is

$$
V(z)=\frac{1}{2} k z^{2}+\tau \lambda(z-\varphi g)
$$

where setting $k=m \omega^{2}, \tau=\sqrt{m \omega / \hbar}(\hbar \omega / 2), \varphi=\sqrt{\hbar / m \omega}$ and substituting (43) into (42), we can write classical $\kappa(y)$ to simply produce

$$
\kappa(y)=\Phi \int_{-\infty}^{\infty}\left(\frac{1}{2} k z^{2}+\tau \lambda(z-\varphi g)\right) e^{-\alpha(z-y)^{2}} d z,
$$

where $\alpha=6 m / \beta \hbar^{2}, \Phi=\sqrt{6 m / \beta \pi \hbar^{2}}$. The final the pathintegral method is

$$
\kappa(y)=\frac{\Phi \sqrt{\pi}\left(k+2 k y^{2} \alpha+4 \alpha \lambda \tau(y-\varphi g)\right)}{4 \alpha^{3 / 2}} .
$$

In such case it is more useful to write the partition function of $\kappa(y)$ as

$$
e^{-\beta F_{c l-K}}=\Omega \int_{-\infty}^{\infty} e^{-\beta \kappa(y)} d y
$$

where $\Omega=m / 2 \pi \hbar U$. Substituting (45) into (46) can obtain the partition function of path-integral method $(\kappa(y))$ in case of harmonic oscillator asymmetric potential

$$
\begin{aligned}
e^{-\beta F_{c l-K}}= & \Omega \int_{-\infty}^{\infty} e^{-\beta\left(\Phi \sqrt{\pi}\left(k+2 k y^{2} \alpha+4 \alpha \lambda \tau(y-\varphi g)\right) / 4 \alpha^{3 / 2}\right)} d y \\
= & \Omega e^{-\left(\left(\beta \sqrt{\pi} \Phi k / 4 \alpha^{3 / 2}\right)-\left(4 g \beta \alpha \tau \lambda \varphi / 4 \alpha^{3 / 2}\right)\right)} \\
& \times \int_{-\infty}^{\infty} e^{-\beta \sqrt{\pi} \Phi\left(\left(2 k \alpha y^{2}+4 \alpha \tau \lambda y\right) / 4 \alpha^{3 / 2}\right)} d y, \\
e^{-\beta F_{c l-K}}= & \frac{\sqrt{2} \sqrt[4]{\pi} \Omega}{\sqrt{\beta k \Phi / \sqrt{\alpha}}}\left[e^{-\left(\beta \Phi \sqrt{\pi} / 4 k \alpha^{3 / 2}\right)\left(k^{2}-2 \alpha \lambda^{2} \tau^{2}-4 g k \alpha \lambda \tau \varphi\right)}\right] .
\end{aligned}
$$

Substituting $\alpha=6 m / \beta \hbar^{2}, k=m \omega^{2}, \tau=\sqrt{m \omega / \hbar}(\hbar \omega / 2)$, $\varphi=\sqrt{\hbar / m \omega}, \Phi=\sqrt{6 m / \beta \pi \hbar^{2}}$, and $\Omega=\sqrt{m / 2 \pi \hbar U}$ into (47) leads to

$$
\begin{aligned}
e^{-\beta F_{c l-K}} & \\
= & \frac{1}{\hbar \beta \omega} \\
& \times e^{-\left(\beta^{2} \hbar^{2} / 24 m^{2} \omega^{2}\right)\left(m^{2} \omega^{4}-\left(3 \lambda^{2} m^{2} \omega^{3} / \beta \hbar\right)-\left(12 g \lambda m^{2} \omega^{2} / \beta \hbar\right)\right)}, \\
& -\beta F_{c l-K}=-\ln (2 f)-\left(\frac{f^{2}}{6}-\frac{f \lambda^{2}}{4}-g f \lambda\right) .
\end{aligned}
$$

Accordingly, the Helmholtz free energy the path-integral method $(\kappa(y))$ is given by

$$
F_{c l-K}(f)=\frac{1}{\beta}\left[\ln (2 f)+\left(\frac{f^{2}}{6}-\frac{f \lambda^{2}}{4}-g f \lambda\right)\right] .
$$

TABLE 1: Comparison of the Helmholtz free energy of the harmonic oscillator asymmetric potential obtained from Feynmans approach $\left(G_{\text {new }}\right)$, path-integral method $\kappa(y)\left(G_{c l-K}(T)\right)$, where $\lambda$ is the value of asymmetric potential, $g$ is the value of axes-shift potential system, and $T$ is the temperature, setting $g=\lambda=1$.

\begin{tabular}{lccc}
\hline$T(K)$ & $G_{\text {new }}(T)$ & $G_{c l-K}(T)$ & $\%$ Difference \\
\hline 1 & -1.167350290774 & -1.166666666667 & 0.058579189 \\
2 & -3.981008518269 & -3.980922055573 & 0.002171902 \\
3 & -7.813921629128 & -7.813895954231 & 0.000328579 \\
4 & -12.319532395568 & -12.319521555626 & 0.000087989 \\
5 & -17.327718009705 & -17.327712457674 & 0.000032041 \\
6 & -22.737227955451 & -22.737224741848 & 0.000014133 \\
7 & -28.480839348833 & -28.480837324869 & $7.10640 \times 10^{-6}$ \\
8 & -34.510649356211 & -34.510648000211 & $3.92922 \times 10^{-6}$ \\
9 & -40.790784085205 & -40.790783132793 & $2.33487 \times 10^{-6}$ \\
10 & -47.293369220882 & -47.293368526548 & $1.46814 \times 10^{-6}$ \\
11 & -53.996120765666 & -53.996120243988 & $9.66139 \times 10^{-7}$ \\
12 & -60.880815552301 & -60.880815150468 & $6.60032 \times 10^{-7}$ \\
13 & -67.932273353648 & -67.932273053847 & $4.65256 \times 10^{-7}$ \\
14 & -75.137653101331 & -75.137652848275 & $3.36789 \times 10^{-7}$ \\
15 & -82.485950683258 & -82.485950477511 & $2.49433 \times 10^{-7}$ \\
\hline
\end{tabular}

After introducing new variable of the Helmholtz free energy path-integral method $(\kappa(y))$ as $G_{c l-K}=2 F_{c l-K} / \hbar \omega, f=$ $\hbar \omega \beta / 2$, the Helmholtz free energy of the path-integral method $(\kappa(y))$ is then transformed to

$$
G_{c l-K}(f)=\frac{1}{f} \ln (2 f)+\frac{f}{6}-\frac{\lambda^{2}}{4}-g \lambda .
$$

This is known as the Helmholtz free energy of the pathintegral method $(\kappa(y))$. We can calculate numerical of the Helmholtz free energy Feynmans $\left(G_{\text {new }}(f)\right.$ approach in Section 3) and the Helmholtz free energy of the path-integral method $(\kappa(y))\left(G_{c l-K}(f)\right.$ in Section 4) for the harmonic oscillator asymmetric potential by mathematica program to give Tables 1 and 2 as shown in Section 5.

\section{The Numerical Result}

In this section, we have evaluated numerical of the Helmholtz free energy Feynmans $\left(G_{\text {new }}(f)\right)$ approach and the Helmholtz free energy of the path-integral method $(\kappa(y))\left(G_{c l-K}(f)\right)$ for the harmonic oscillator asymmetric potential is (39) and (50). By substituting $f=\hbar \omega / 2 k_{B} T$ into (39) and (50), we obtain the Helmholtz free energy Feynmans $\left(G_{\text {new }}(T)\right)$ approach as follows:

$$
G_{\text {new }}(T)=\frac{2 k_{B} T}{\hbar \omega} \ln \left(2 \sinh \left(\frac{\hbar \omega}{2 k_{B} T}\right)\right)-\frac{\lambda}{4}(\lambda+4 g)
$$

and the Helmholtz free energy of the path-integral method $(\kappa(y))\left(G_{c l-K}(T)\right)$

$$
G_{c l-K}(T)=\frac{2 k_{B} T}{\hbar \omega} \ln \left(\frac{\hbar \omega}{k_{B} T}\right)+\frac{\hbar \omega}{12 k_{B} T}-\frac{\lambda^{2}}{4}-g \lambda .
$$

For the beginning of the numerical shooting method, we need to input the parameters $\lambda, g$, and $T$ and (39) and (50) into 
TABLE 2: Comparison of the Helmholtz free energy of the harmonic oscillator asymmetric potential obtained from Feynmans approach $\left(G_{\text {new }}\right)$, path-integral method $\kappa(y)\left(G_{c l-K}(T)\right)$, where we may vary the value of $g, \lambda, T$.

\begin{tabular}{lccccc}
\hline$\lambda$ & $g$ & $T(K)$ & $G_{\text {new }}(T)$ & $G_{c l-K}(T)$ & $\%$ Difference \\
\hline-1 & 1 & 1 & 0.832649709225 & 0.833333333333 & 0.082068555 \\
-2 & 2 & 2 & 0.268991481731 & 0.269077944427 & 0.032138119 \\
-3 & 3 & 3 & 0.186078370872 & 0.186104045769 & 0.013796943 \\
-4 & 4 & 4 & 0.930467604432 & 0.930478444374 & 0.001164993 \\
-5 & 5 & 5 & 2.672281990295 & 2.672287542326 & 0.000207763 \\
-6 & 6 & 6 & 5.512772044548 & 5.512775258152 & 0.000058294 \\
-7 & 7 & 7 & 9.519160651167 & 9.519162675130 & 0.000021262 \\
-8 & 8 & 8 & 14.739350643789 & 21.739351999789 & $9.199869 \times 10^{-6}$ \\
-9 & 9 & 9 & 21.209215914795 & 28.956631473452 & $2.490557 \times 10^{-6}$ \\
-10 & 10 & 10 & 28.956630779118 & 38.003879756012 & $2.397841 \times 10^{-6}$ \\
-11 & 11 & 11 & 38.003879234334 & 48.369184849532 & $1.372696 \times 10^{-6}$ \\
-12 & 12 & 12 & 48.369184447699 & 60.067726962410 & $8.307624 \times 10^{-7}$ \\
-13 & 13 & 14 & 60.067726646352 & 73.112347151725 & $5.261694 \times 10^{-7}$ \\
-14 & 14 & 15 & 73.112346898668 & 87.514049522489 & $3.461208 \times 10^{-7}$ \\
-15 & 15 & 87.514049316743 & $2.351017 \times 10^{-7}$ \\
\hline
\end{tabular}

the mathematical program [9]. Next, we obtain the numerical values of $G_{\text {new }}(T)$ and $G_{c l-K}(T)$ for the harmonic oscillator asymmetric potential as displayed in Tables 1 and 2 .

From Table 1 we show that magnitude of the Helmholtz free energy decrease from -1.1673 to -82.4859 with increasing the temperature of system. From Table 2 we illustrate magnitude of the Helmholtz free energy increase from 0.8326 to 87.5140 with increasing the temperature of system.

Nevertheless, $G_{c l-K}(T)$ is not as useful as Tables 1 and 2 might indicate. The first, it can not be used when quantum mechanics exchange effect exist. The second, it fails in its present form when the potential $V(y)$ has a very large derivative as in the case of hard-sphere interatomic potential (see Lennard-Jones potential [10]).

\section{Conclusion}

This work is interested in finding the canonical density matrix by Feynman's technique and kinetic energy per unit length and evaluation of the Helmholtz free energy. The results show that the magnitude of the density matrix $\left(\widehat{\mathscr{P}}_{u}\right)$ and the kinetic energy per unit length $\left(\widehat{\tau}_{L}\right)$ vary according to the parameters $\lambda, g$, and $T$, respectively. The magnitude of $\left(\widehat{\mathscr{P}}_{u}\right)$ and $\left(\widehat{\tau}_{L}\right)$ for these cases $\lambda$ is positive with the graph of $\left(\widehat{\mathscr{P}}_{u}\right)$ and $\left(\widehat{\tau}_{L}\right)$ moving from right hand side to left hand side. Moreover, we found that as the values of $g$ have increased and the amplitude of $\left(\widehat{\mathscr{P}}_{u}\right)$ and $\left(\widehat{\tau}_{L}\right)$ has decreased.

\section{Conflict of Interests}

The authors declare that there is no conflict of interests regarding the publication of this paper.

\section{Acknowledgments}

Piyarut Moonsri and Artit Hutem wish to thank the Institute Research and Development, Chemistry Division and Physics
Division, Faculty of Science and Technology, Phetchabun Rajabhat University, Thailand. This work is supported by the Institute Research and Development, Phetchabun Rajabhat University.

\section{References}

[1] N. H. March and A. M. Murray, "Relation between Dirac and canonical density matrices, with applications to imperfections in metals," Physical Review Letters, vol. 120, no. 3, pp. 830-836, 1960.

[2] I. A. Howard, A. Minguzzi, N. H. March, and M. Tosi, "Slater sum for the one-dimensional $\operatorname{sech}^{2}$ potential in relation to the kinetic energy density," Journal of Mathematical Physics, vol. 45, no. 6, pp. 2411-2419, 2004.

[3] N. H. March and L. M. Nieto, "Bloch equation for the canonical density matrix in terms of its diagonal element: the Slater sum," Physics Letters A: General, Atomic and Solid State Physics, vol. 373, no. 18-19, pp. 1691-1692, 2009.

[4] R. K. Pathria, Statistical Mechanics, Butterworth Heinemann, Oxford, UK, 1996.

[5] G. B. Arfken and H. J. Weber, Mathematical Methods for Physicists, Elsevier Academic Press, New York, NY, USA, 6th edition, 2005.

[6] K. F. Riley and M. P. Hobson, Mathematical Method for Physics and Engineering, Cambridge University Press, 3rd edition, 2006.

[7] R. P. Feynman, Statistical Mechanics, Addison-Wesley, London, UK, 1972.

[8] I. N. Levin, Quantum Chemistry, Pearson Prentice Hall, 6th edition, 2009.

[9] R. L. Zimmerman and F. I. Olness, Mathematica for Physics, Addison-Wesley, Reading, Mass, USA, 2nd edition, 2002.

[10] A. Hutem and S. Boonchui, "Numerical evaluation of second and third virial coefficients of some inert gases via classical cluster expansion," Journal of Mathematical Chemistry, vol. 50, no. 5, pp. 1262-1276, 2012. 

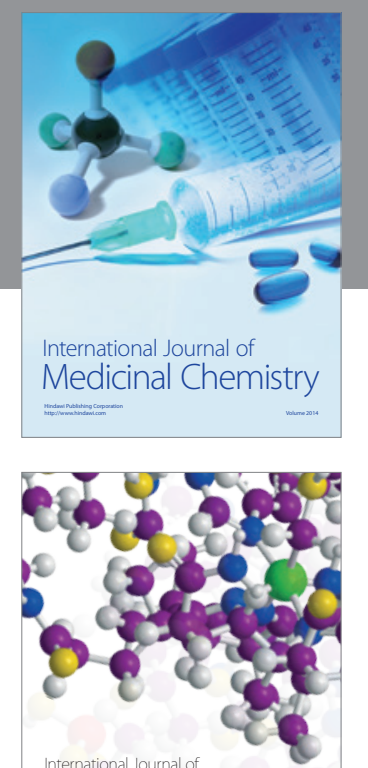

\section{Carbohydrate} Chemistry

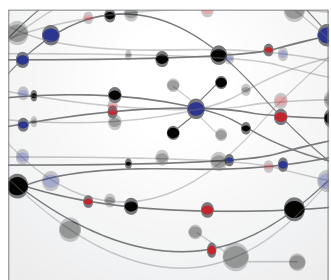

The Scientific World Journal
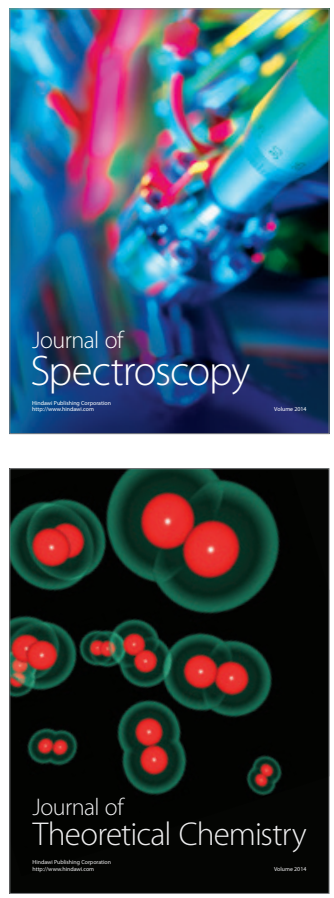
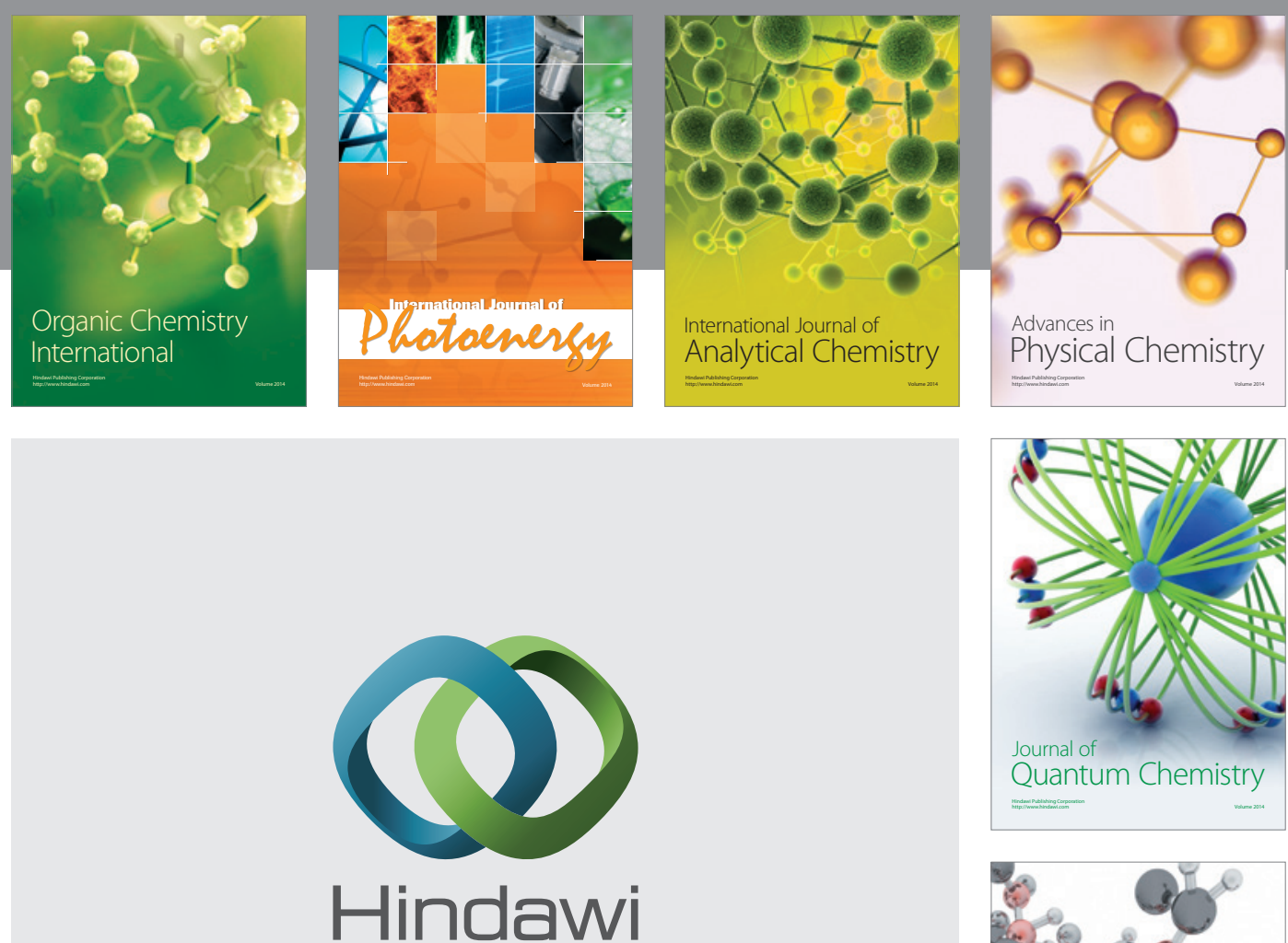

Submit your manuscripts at

http://www.hindawi.com

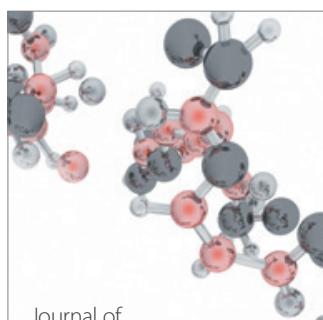

Analytical Methods

in Chemistry

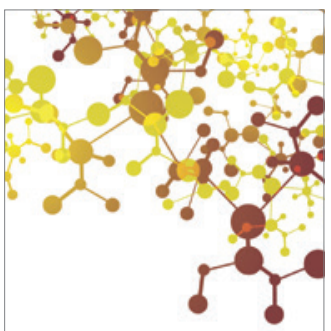

Journal of

Applied Chemistry

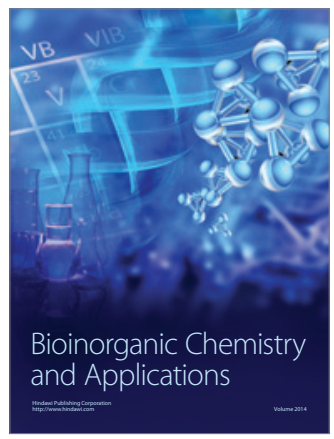

Inorganic Chemistry
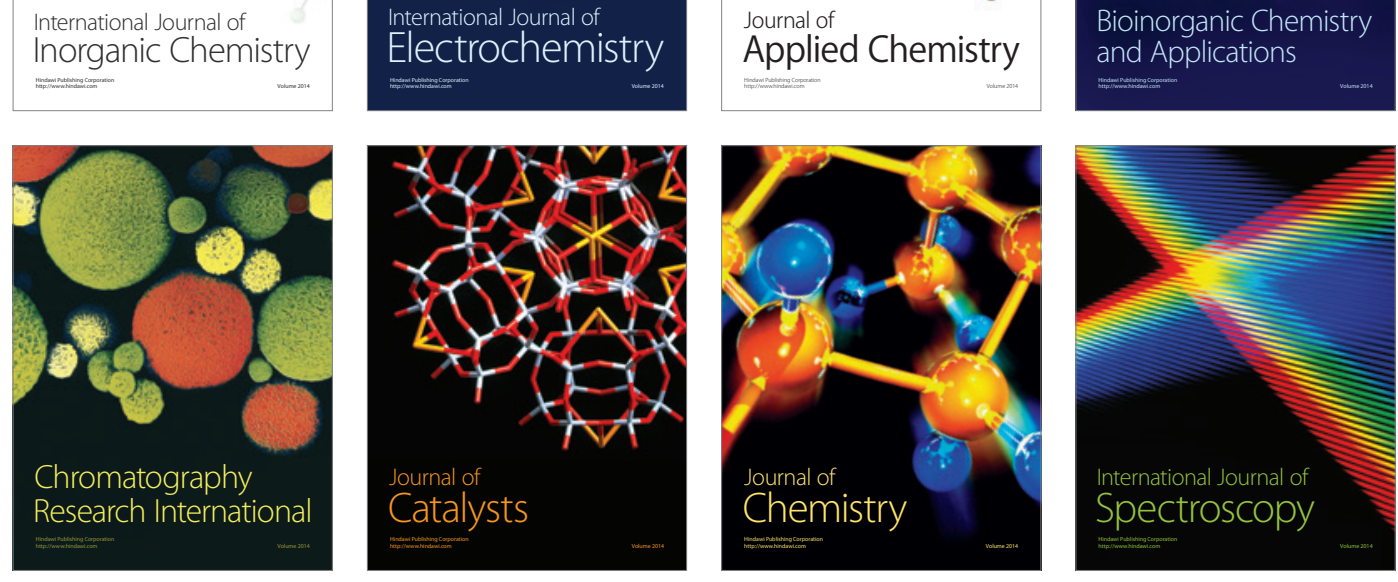